Ford and Clarke present evidence of clonal proliferation in primary reticular neoplasms (compare chapter by Court Brown and Tough in Advances).

Another topic of present interest in cancer research is that of cellular interactions. This section deals with cellular interactions in vitro, describes an attempt to determine the time of mitosis in relation to ribonucleic acid and deoxyribonucleic synthesis and contains a discussion of a subcellular agent which produces ascites tumours in mice. Daoust has contributed a chapter on pre-neoplastic liver parenchyma in which the idea again emerges that changes in nucleic acids may represent essential steps in carcinogenesis. The section is concluded with a diseussion of cell interactions by Weiss.

Immunological aspects of cancer research are considered in seven chapters which deal with acquired immunological tolerance, auto-antibody formation, immunity to virusinduced tumours, etc., and, in the final section, chemotherapy, mechanisms of resistance to antimetabolites are described as well as cross-resistance. In the final chapter Burchenal surveys the problems in clinical resistance.

These two volumes from Academic Press are valuable additions to their respective series. They seem to point the way to proliferation of research in the region of chromosomal and nucleic acid abnormalities which may prove to be at the very root of the carcinogenic process.

W. J. P. NEISH

\section{DETECTING BLOOD DISORDERS}

Atlas de Thrombodynamographie

Par G. Marehal, M. E. Leroux et M. Samama. Pp. 186. (Paris: Service de Propagande, Édition, Information, 1962.) 60 N.F.

THE authors have produced a comprehensive atlas to illustrate the various changes they can measure in certain blood disorders using the thrombo-elastograph as designed by Dr. Hartert.

This ingenious piece of apparatus records on film the oscillations of a light beam reflected from a mirror mounted on a torsion wire. On this wire is suspended a plunger which is immersed in whole blood or plasma contained in a stainless steel cuvette. The latter oscillates regularly $5^{\circ}$ on either side of the midline, and as the blood or plasma clots naturally or aftor the addition of calcium, the fibrin formed grips the plunger and so transmits the movements of the cuvette to the film. The trace on the film records the time it takes for fibrin to appear first, the rate of fibrin formation and the gripping force of the fibrin formed.

The details of the apparatus and the technique of operat. ing are described in detail together with a useful description of artefacts that may be encountered. The sequence of blood coagulation and the role of each plasma factor and platelet function are discussed and illustrated. Tracings to show the different patterns obtained in certain disorders are also reproduced, namely, thrombocytopenia, abnormalities of platelet function, fibrinogen deficiency, plasma coagulation factor deficiencies, circulating anticoagulants, prothrombin deficiency, fibrinolysis, hypercoagulable states, and anticoagulant-induced defects. The authors illustrate and describe adaptation of the thrombo-elastograph technique to define specific coagulation defects and also the use of this machine in defining the changes in the blood following open-heart surgery.

There is a very comprehensive text accompanying the illustrations, and the publishers are to be congratulated on printing this in French, English and German. It is a pity that more care has not been taken with the English text, which is quaint and in parts frankly misleading due to the translation being too literal. Further, the authors can be criticized for trying to convert this into an incomplete text-book on hæmorrhagic diseases.
A shorter text confined specifically to the problems and value of thrombo-elastography would have been more acceptable. An unusual feature is the mixture of advertisements with the bibliography, which is incomplete. Authors' names in the text are not designated by the dates of their articles and some authorities quoted in the text are not mentioned in the list of references.

Although there are these defects, this book should be read by those who have, or contemplate having, this instrument to assist in the problems of routine diagnosis or for research purposes.

A. A. SharP

\section{SOUTHERN NIGERIAN ANTHROPOMETRY}

The Physical Anthropology of Southern Nigeria A Biometric Study in Statistical Method. By the late P. A. Talbot and H. Mulhall. (Occasional publications of the Cambridge University Museum of Archæology and Ethnology.) Pp. xvi + 127. (Cambridge: The University Press, 1962.) 32s. 6d.

HIS monograph was made possible by the late Dr.

P. Amaury Talbot, former resident, Civil Administration, Southern Nigeria, who for thirty years collected anthropometric and anthroposcopic data in this region which he administered. After his death, Mrs. Talbot put the data at the disposal of the Duckworth Laboratory of Physical Anthropology, Cambridge, the director of which, Dr. J. C. Trevor, further sought the statistical help of Dr. Mulhall. It has been the latter author's task to reduce the mass of undigested data to the meticulous and precise analysis seen in this published work.

The first chapter is understandably devoted to previous regional classification of Southern Nigeria and the relevant population numbers (although, incidentally, the sample sizes utilized in the analysis are not relative to the population totals for the regional groups). This is followed by a diversion into statistical procedures, Chapters 2-7, which are meant as a general introduction to anthropometric statistics, as well as to the final detailed analysis. Mulhall is to be congratulated on the way in which he carefully takes us through the statistical methods; problems of sampling, probability and theoretical distribution models, statistical relationships, and problems of group divergence.

The original contribution of this book is in its analysis of the measurements of nearly 7,000 inhabitants from more than 50 groups. Although there is some debate as to the relative merits of an exhaustive analysis of a few dimensions (12 in this case), as opposed to the lessdetailed analysis of more measurements, one is left wishing that a few more had been available.

This aralysis of the Nigerian data forms the last two chapters. First, the body measurements are considered, culminating in the computation of $D^{2}$ values for all the regional groups. This is followed by a consideration of skin colour (alas, Talbot was recording in the days before colorimetric scales and the portable spectrophotometer were in use). This pigmentary information is a useful addition to the study, and in part corroborates and in part challenges the conclusions drawn from the metrical investigation. Finally, there is reclassification of the groups analysed.

As Trevor and Mulhall intended, this is a survey of statistical methods in some aspects of physical anthropology, combined with a detailed analysis of data from one area of West Africa-a s omewhat unique combination.

A final chapter reviewing the results of this analysis in relation to other human biological findings from Nigeria (serological, dermatoglyphic, etc.) would have been a very useful addition. Also, perhaps some mention could have been made of possible environmental differences 